\title{
Charge transport characterization of the alternative low power hybrid engine (alphie) with particle-in-cell simulations
}

D. Dyubo, ${ }^{1}$ J. Gonzalez, ${ }^{2}$ O. Tsybin, ${ }^{1}$ and L. Conde ${ }^{*, 3}$

1) Institute for Physics, Nanotechnology and Telecommunications. Peter the Great St. Petersburg Polytechnic University. Polytechnichkaya 29, 195221 St. Petersburg, Russia

2) DIFFER-Dutch Institute for Fundamental Energy Research. De Zaale 20, 5612 AJ, Eindhoven,

The Netherlands.

${ }^{3)}$ Departamento de Física Aplicada. E.T.S.I. Aeronáutica y del Espacio. Universidad Politécnica de Madrid. Cardenal Cisne 28040 Madrid, Spain.

(*Corresponding author: luis.conde@upm.es)

(Dated: 30 September 2021)

The Alternative Low Power Ion Engine (alphie) is a high specific impulse plasma thruster where both electror ions flow combined through the open spaces of its two-grids system. Ionizing electrons from its external cathode in towards the ionization chamber accelerated by the potential drop established between the grids whereas ior towards the opposite direction and are later neutralized by electrons emitted from the cathode. In this configuratic ion current is not space-charge limited, contrary to conventional gridded ion engines where only ions are trans] through the grids. Since previous approaches that consider only one charged species are not valid for the alphi grids system, the characterization of geometrical parameters, is studied using a fully particle-in-cell (PIC) simul This numerical scheme calculates the velocities and positions of electrons and ions within a structured axial-symı mesh, which mimics one hole of the grids. The charged particle densities, currents as well as their axial and radial velocities along the simulation domain are evaluated. Additionally, important reductions in the computationa have been obtained using different time scales for ions and electrons. Numerical results show that moderate ch in the thickness and separation of the grids with respect to the values employed in the prototype currently tested laboratory do not significantly affect the exhaust ion current or the ion beam collimation. Since the average velo the axial ions is essentially insensitive to small modifications of the geometric parameters, so will the specific in and thrust delivered.

\section{INTRODUCTION}

Electrostatic plasma thrusters (EPT) for in-space propulsion exhaust a high velocity ion current to generate impulse. A jet of electrons and ions, usually denominated plasma plume, is produced when electrons are added to the ion beam to neutralize the space-charge effects ${ }^{1-3}$

Plasma thrusters convert electric power into kinetic energy of ions that are accelerated to $v_{e x} \sim 10-40 \mathrm{~km} / \mathrm{s}$ range exhaust velocities, one order of magnitude higher than those achieved by propellants in conventional chemical thrusters. This gives high specific impulses $I_{s p}=v_{e x} / g_{o}$, where $g_{o}$ is the standard Earth's gravity. However, ion mass production rates $\dot{m}_{i}$ are low and delivered thrusts $F=\dot{m}_{i} v_{e x}$ are then limited. Electrostatic plasma thrusters are therefore characterized by high specific impulses and low thrusts ${ }^{1-3}$.

Most plasma thrusters require electron sources, such as hollow cathodes for both, ionization of the propellant gas and ion beam neutralization. Hall effect thrusters (HET) and the different multi-cusp field thrusters (MCFT) accelerate heavy ions in the self-consistent electric field created in a plasma discharge inside annular or cylindrical ionization chambers where ionizing electrons are confined by different magnetic field configurations. The physical processes involved in positive charge acceleration and propellant gas ionization are closely related in HET and MCFT thrusters ${ }^{1-3}$.

Gridded ion engines (GIE) produce the electrostatic acceleration of ions extracted from a plasma source and require two electron sources. A first hollow cathode is located inside the ionization chamber to produce the ionization of the prop neutral gas by electrons. The ions are extracted and ac ated using a system of two or three electrically biased aperture grids (plane or dish-shaped) denominated as tl optics $^{1-3}$.

The first screen grid is in contact with the plasma insi ionization chamber, it extracts the ions and repels the trons back. Extracted positive charges are accelerated electric field established between this first screen and th ond acceleration grid to produce the main part of the Finally, the ion beam is neutralized by electrons from a s hollow cathode arranged in front of the ion optics.

An additional third grid biased at a low negative poter usually located in front of the acceleration mesh to avo backflow of electrons from the neutralizer cathode. This tional mesh shields the acceleration grid from ion bon ment of charge-exchange ions and potentially has a lifetime and reduced sputtering compared with that of grid systems ${ }^{1,4}$.

The inter-grid spacing in GIEs is small (typically $1 \mathrm{n}$ produce high electric fields that increase the velocity o These small distances combined with high voltages can electrical breakdown and shorting. Therefore, this sens to small geometrical changes of ion optics system is an

Contrary to HET and MCFT concepts, the ion pr tion and its acceleration are independent processes in since only positive charged particles move through the Thus, the maximum ion current density transported thro bounded by space-charge effects ${ }^{1,4}$. 


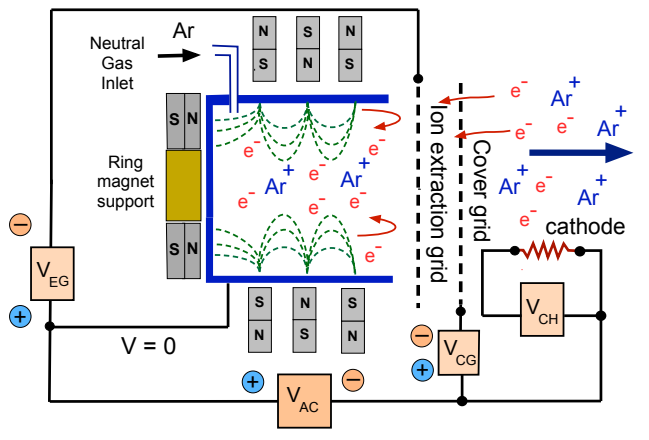

FIG. 1. Diagram of the alphie plasma thruster cross section and its electrical connections with Argon as propellant gas. The dotted curves represent the magnetic field lines.

The Alternative Low Power Ion Engine (alphie) is a new gridded plasma thruster recently introduced in Refs. 5-9. It is aimed to provide mid-sized satellites of $100-300 \mathrm{~kg}$ in low Earth orbit high specific impulses with thrusts within the 0.1$10 \mathrm{mN}$ range at reduced power consumption $(<300 \mathrm{~W})$ and low propellant mass fluxes $(<1 \mathrm{sccm})$ of Argon and Xenon. The measured average ion exhaust speeds $37-44 \mathrm{~km} / \mathrm{s}$ of Argon ions give typical specific impulses $I_{s p}=v_{e x} / g_{o}$ within the $3,500-4,400 \mathrm{~s}$ range, higher than low power Hall effect thrusters ${ }^{8}$.

Its two-grids system operates differently from the usual GIE scheme described above. In the alphie, electrons and ions flow through the open spaces of its two flat parallel grids whereas only ions move through the usual GIE ion optic system. Charges are accelerated by the self-consistent electric field created by the currents of electrons and ions in addition to the electric potential applied to the grids. Consequently, the exit ion beam is not space-charge limited. This basic alphie operation scheme has been investigated in the laboratory 7,8 and it was also confirmed by particle in cell (PIC) numerical simulations ${ }^{9}$.

Computer modelling of propulsive systems is essential to characterize their performance and to improve designs, as it saves time and resources ${ }^{9-12}$. However, numerical schemes aimed at optimizing the geometry of conventional GIE ion optics that only consider the transport of ions are not valid for this new alphie concept.

Alternatively, the counterflow of positive and negative charged particles has been previously studied using PIC codes that generally require significant computational resources ${ }^{12-17}$. Specifically, characterization studies of the alphie two-grids system require improved codes to reduce the time consumed in calculations within affordable limits, while holding the number of simulation particles high enough to obtain realistic conclusions.

In the following we discuss the geometrical characterization of the alphie two-grids system using a particle-in-cell (PIC) numerical scheme. The simulations reproduce the trans-

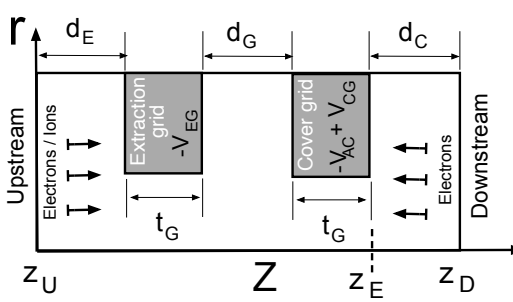

FIG. 2. The axial-symmetric simulation domain of two aligned The ionization chamber electrically biased at zero potential is 1 upstream at $z<z_{U}$ and the cathode is located at the $z>z_{D}$ stream side. The coordinate $z_{E}$ indicates the exit section of the grid.

port in the self-consistent electric field of charged pa through a hole of the grids using a structured axial-sym mesh. The particle properties such as charge and veloci weighted in the mesh nodes, and later are used to evalua local self-consistent electric field. As we shall see, no : icant changes in the exit ion current or in the collimat the beam due to small changes in the geometric parame grids are produced.

This paper is organized as follows; the alphie $\mathrm{p}$ thruster conception is briefly discussed in section II. T tails of the PIC numerical scheme employed to study the tron and ion counterflow between the electrically biasec and the computational domain is introduced in sections I IV. The performance for a range of geometrical parame the two-grid systems is discussed in section $\mathrm{V}$ and fina end with some concluding remarks.

\section{THE ALPHIE PLASMA THRUSTER}

The schematic cross section of alphie thuster and it trical connections are shown in Fig. 1 and details of $i$ eration and performance in laboratory tests can be fol references 5-9.

Briefly, the cathode arranged in front of the grids pro electrons for both ionization and ion beam neutralization ilarly to HET and MCFT configurations. The Argon ior downstream through the flat extraction and cover grid are electrically biased. Typically, the extraction grid po is $V_{E G} \simeq-150 \mathrm{~V}$ and the cover grid potential $V_{C G}$ is $\mathrm{u}$ null to maximize the ion output current ${ }^{7}$.

A fraction of electrons from the cathode move in through the open spaces of the grids. Inside the plasma ization chamber, these electrons have energies in the ra $400-500 \mathrm{eV}$ energized by the $V_{A C}=400-750 \mathrm{~V}$ accele potential and are confined by the magnetic field lines (c lines in Fig. 1). This high energy electron group ioni neutral gas introduced in the plasma chamber.

Positive ions are attracted towards the extraction gri move outwards accelerated by the self-consistent $\mathrm{e}$ field. They reach final exit velocities in the $40-50 \mathrm{~km} / \mathrm{s}$ 


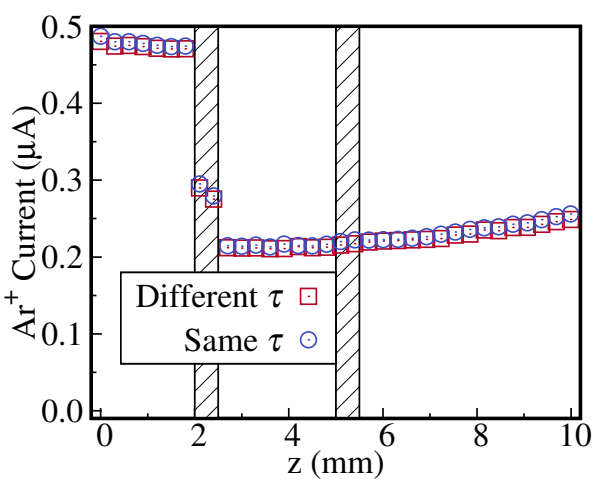

(a) The ion current $I_{i}(z)$.

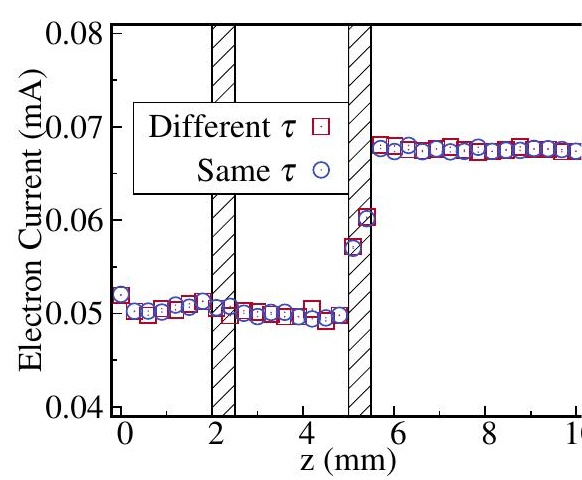

(b) The electron current $I_{e}(z)$.

FIG. 3. Comparison of electron and ion currents flowing through the simulation domain of Fig. 2 calculated with equal iteration tin $10^{-11} \mathrm{~s}$ (open circles) and when it is increased to $\tau=10^{-9} \mathrm{~s}$ for the ions (open squares).

controlled also by the $V_{A C}$ acceleration voltage ${ }^{7,8}$. In the stationary state, this ion current is not space-charge limited since both, positive and negative charges move through the alphie two-grid system. Finally, the positive charge outflow is neutralized by electrons from the cathode placed in front of the grids to form a quasi-neutral plasma plume.

Therefore, the alphie thruster can be categorized as an hybrid concept as the two grids focus and accelerate the electric charges similarly to gridded ion engines, however it only needs one external cathode, as with HET and MCFT plasma thrusters.

\section{THE NUMERICAL SIMULATION DOMAIN}

The grids in the alphie prototype currently tested in the laboratory are two stainless steel disks with 665 aligned holes of $1 \mathrm{~mm}$ in diameter drilled within a circle of $20 \mathrm{~mm}$ in radius. Fig. 2 represents schematically the axial-symmetric simulation domain of two aligned holes where $t_{G}$ is its thickness and $d_{G}$ their separation. In our numerical simulations, the position of the first extraction grid is fixed at $d_{E}=2.0 \mathrm{~mm}$ of the upstream boundary at $z_{U}=0.0 \mathrm{~mm}$ and the ion downstream exit section $z_{D}$ is located at the $d_{C}=5.0 \mathrm{~mm}$ distance from the cover grid. The end of the cover grid is located at $z_{E}=d_{E}+d_{G}+2 t_{G}$ as shows Fig. 2. The metallic ionization chamber of Fig 1 is considered at zero voltage $(V=0)$ and it is also the reference for the electric potentials applied to the grids.

In the current prototype $d_{G}=2.0 \mathrm{~mm}, t_{G}=0.5 \mathrm{~mm}$ and these values will be used in the following as a reference. These geometrical parameters will be modified in the following to study their impact in the electron and ion currents transported through the two-grids system.

As shown in Fig. 2, the upstream region $z<d_{E}$ represents the ionization chamber of the scheme in Fig. 1 whereas the downstream side is the vacuum in front of the cover grid. The grids are typically biased to $-150 \mathrm{~V}$ and $-600 \mathrm{~V}$ with $\mathrm{r}$ to the upstream boundary kept at $0 \mathrm{~V}$. Typical thruste rents in the experiments are $I_{E} \simeq 100-500 \mathrm{~mA}$ that $150-750 \mu \mathrm{A}$ transported through the computational $\mathrm{d}$ of Fig. 2.

The boundary conditions are similar to those of R The electric potential at $z_{U}$ is assumed as null to mat ionization chamber potential and the axial electric field $i$ at the opposite side $z_{D}$, in the distant quasineutral plasn the maximum radial position and along the axis of sym radial component of the electric field is considered as $n$

Electrons from the cathode enter the downstream $z=z_{D}$ of the computational domain and ions and electro ter from the upstream ionization chamber. Two populati electrons coexist in the $z<d_{E}$ region confined by the ne potential of the extraction grid and the magnetic field The fast electrons from the cathode and the slow gro sulting from the impact ionization of neutral atoms ups Only electrons from the cathode and exiting ions are $\mathrm{p}$ on the downstream side.

The initial electron and ion temperatures, densities a locities in the simulation domain were selected to matc ical plasma conditions. Electrons from the cathode are duced at the downstream side with a temperature of 2 and axial mean velocity of $-87 \mathrm{~km} / \mathrm{s}$. Secondary ele from ionization processes at the plasma chamber have a axial velocity of $87 \mathrm{~km} / \mathrm{s}$ and a temperature of $500 \mathrm{~K}$ ions enter upstream in the simulation region with a me: locity of $300 \mathrm{~m} / \mathrm{s}$ and a temperature of $500 \mathrm{~K}$. The $t$ reference density for both species is in the order of $10^{1}$ giving a Debye length of $0.1 \mathrm{~mm}$. From the downstrear of the mesh, a current of $150 \mu \mathrm{A}$ of electrons is injecte at the ionization chamber side currents for ions and ele are one order of magnitude below. Finally, when a ch particle exits the numerical domain or collides with the it is removed from the simulation. 


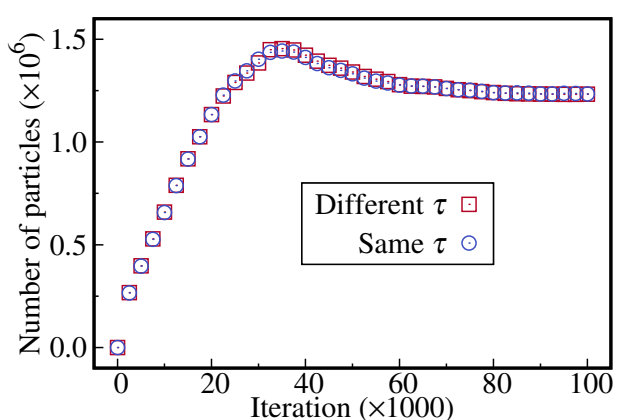

(a) Number of macro-particles

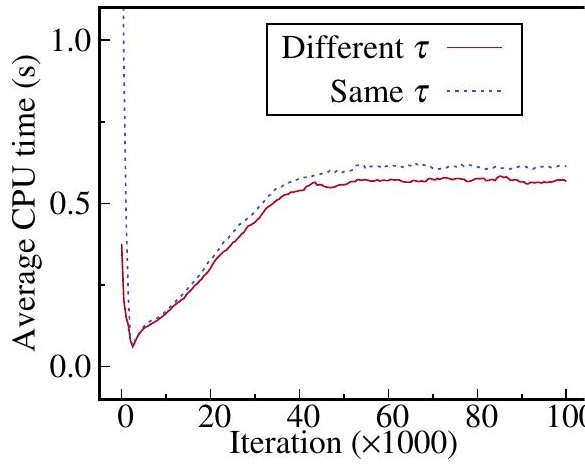

(b) Mean computation time

FIG. 4. Comparison of numerical simulation performance using equal time step of $\tau=10^{-11} \mathrm{~s}$ for ions and electrons (dotted lines) anc it is increased to $\tau=10^{-9} \mathrm{~s}$ for the ions (solid lines).

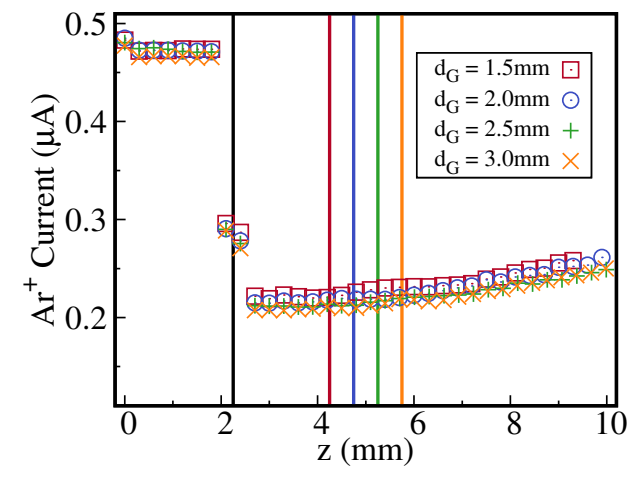

(a) Ion Current $I_{i}(z)$.

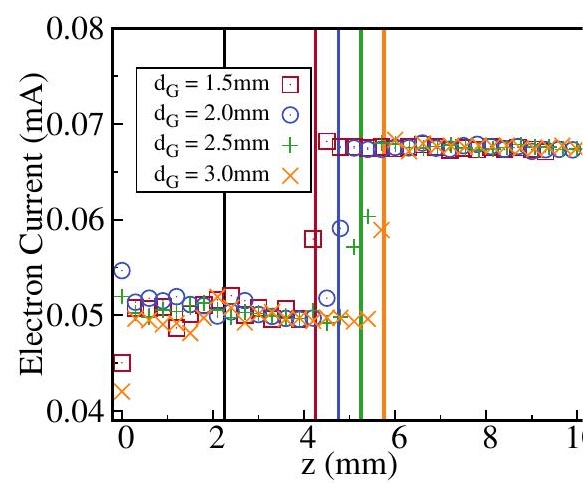

(b) Electron current $I_{e}(z)$.

FIG. 5. The ion and electron current for different distances $d_{G}$ between the extraction and cover grids. The grid thickness remains cons the base case of $t_{G}=0.5 \mathrm{~mm}$. Vertical lines represent the mid position of grids.

\section{THE FPAKC PIC NUMERICAL SCHEME}

The usual simulation scheme where electrons are in thermal equilibrium following a Boltzmann energy distribution and ions are treated as particles is not valid to describe the charged particle counterflow through the alphie's two-grids system. A full particle-in-cell code where both, ions and electrons are considered as macro-particles driven by the local electric field is required to study the charge transport through the simulation domain of Fig. 2.

The numerical scheme employed in our calculations, now named Finite element PArticle Kinetic Code (fpakc), is an evolution of a previous particle-in-cell code discussed in Ref. 9. The current version can tackle different problems by means of an input file and the previous original uniform finite difference grid has been replaced by a finite el mesh. Additionally, the possibility of advancing the ent particle species with specific time scales has bee introduced. These two new capabilities are of major i tance for the grid characterization analysis of alphie th concept. The predictions of previous codes ${ }^{9}$ and fpakc validated against the experimental data of Ref. 7.

The finite element mesh allows adapting the simulati main to different geometries, however, a structured $\mathrm{m}$ still preferred for the present case. The axial-symmetric putational domain of Fig. 2 is divided in a structured $r$ gular mesh of cells of about $0.1 \mathrm{~mm}$ side length.

As with most PIC codes, the fpakc numerical schem for a number of iterations until a quasi-steady state is re: At each iteration, new particles are injected into the si tion domain of Fig. 2 at both boundaries. All the par 


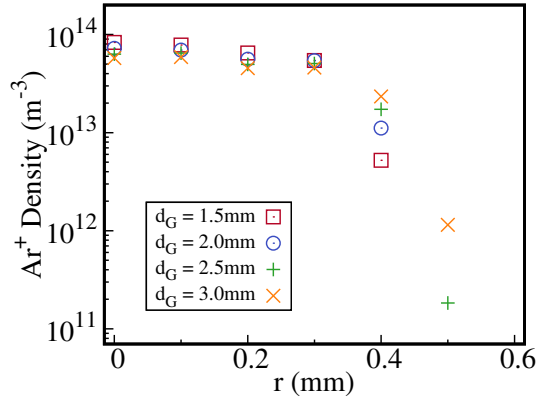

FIG. 6. The axial number density $n_{i}\left(r, z_{E}\right)$ of ions for different distances $d_{G}$ between the extraction and cover grids, holding constant $t_{G}=0.5 \mathrm{~mm}$

old and new are pushed to obtain their new coordinates and velocities. They can move to a different cell or interact with those at adjacent cells, tracked in fpakc by means of a neighbor interaction system. Cells and material surfaces are linked by the nodes they share, reducing the computational cost of assigning particles to specific cells in the simulation. About $10^{6}$ macro-particles are present in the domain when a quasisteady state is reached. Then, the properties of the particles (essentially charge and velocity) are deposited in the nodes of the cells, in which the particles end their motion after each iteration. The self-consistent electric field is computed locally in the finite element mesh by solving the Poisson equation.

The ability to push species with different time scales is essential to reduce computational time. This is a critical issue in situations where both, ions and electrons, are included in the simulation as mobile kinetic species. In the present case, light electrons from the cathode move much faster through the alphie two-grids system than the slow and heavy ions. The multiple time scale feature of the fpakc numerical scheme is illustrated in Figs. 3 and 4.

Figure 3 shows the position of the two grids, which is indicated by the dashed areas. Also, the values of the electron $I_{e}(z)$ and ion $I_{i}(z)$ currents flowing along the computational domain of Fig. 2. Both are evaluated as averages over the velocity distribution function of particles along the cells of the simulation domain.

The current $I_{z}$ is calculated from the axial current density $J_{z}=n v_{z}$ obtained in the structured grid nodes. The integration is approximated by,

$I_{z}=\int_{0}^{r_{\max }} 2 \pi r J_{z}(r) d r \simeq \sum_{j=0}^{5} 2 \pi \frac{r_{j}+r_{j+1}}{2} \frac{J_{z, j}+J_{z, j+1}}{2}\left(r_{j+1}-r_{j}\right)$

where $j$ represents the node along the radial direction.

The calculated Argon ion current per hole remains in Fig. 3a approximately equal for $z<2.2 \mathrm{~mm}$ inside the ionization chamber. After the extraction grid, $I_{i}(z)$ decreases due to the unavoidable losses by the capture of ions on its surface. The ion current remains constant as positive charges gain pos- itive velocity outwards and exit the cover grid. Due self-consistent radial field outside the grid system, iol come even more collimated, resulting in a slight incre current as axial velocity increases. Since the holes o meshes are aligned, ion losses are much lower in the s grid. Due to the self-consistent electric field created $b$ counterflow of charges and the boundary conditions set end of the numerical domain, a slight increment in th rent appears. However, present study concerns the chan the ion distribution at the exit plane $z_{E}<z_{D}$ of the two system in Fig. 2.

The electron current in Fig. 3b follows the opposite Its value is approximately constant for $z>6 \mathrm{~mm}$ in the in front of the cover grid where the cathode is located. trons are absorbed by the cover grid and lost in the simu and consequently, the electron current reduces to rema sentially constant in the inter-grid space and for $z<$ Thus, the electron current becomes more negative in the grids space since electrons are accelerated inwards and takes its minimum value within the ionization chamb cause of the high velocity of ionizing electrons. The ence in magnitude of the electron and ion currents in Fig caused by the high negative velocity of electrons, much than the average ion speed.

Figure 3 shows that numerical results obtained for th and ion $I_{i}(z)$ currents are equal using the equal $\tau=1$ time scale for iteration (open circles) and when it is ince to $\tau=10^{-9} \mathrm{~s}$ for the ions. The number of macro-pa present in the simulation Fig. $4 \mathrm{a}$ is essentially the same a and electrons iterate with the same time step of (open sq or when this time scale is increased for ions by a facto In this last case, the effort required for these calculati Fig. $4 \mathrm{~b}$ shows a reduction of $40 \%$ in the total computa time.

\section{NUMERICAL RESULTS}

We study in first place the effect of the distance $d_{G}$ be the extraction and cover grids, holding constant their thic $t_{G}$. As it was in Fig. 3, the currents $I_{i}(z)$ of ions $\mathrm{m}$ upstream and electrons $I_{e}(z)$ flowing inwards to the ioni chamber are represented in Fig. 5 along the axial direct symmetry.

The solid vertical lines indicate the midpoint posit the two alphie grids for the distances $d_{G}=1.5,2.0,2$. $3.0 \mathrm{~mm}$ for a constant grid thickness of $t_{G}=0.5 \mathrm{~mm}$. T cation of the extraction grid was held fixed and the cove displaced along the axis of symmetry of the simulatic main.

The changes with the distance $d_{G}$ in the electron a currents in Fig. 5 are minimal. Reductions in the di between the grids only produce small increments in th current $I_{i}=I_{i, z}\left(z_{E}\right)$ at the exit section of the cover $\mathrm{g}$ Fig. 5a. We have observed no differences between 2 and $2.0 \mathrm{~mm}$ separation distances. The effect in the el current $I_{e}(z)$ is even smaller as it can be observed in Fi The self-consistent electric field generated between the 


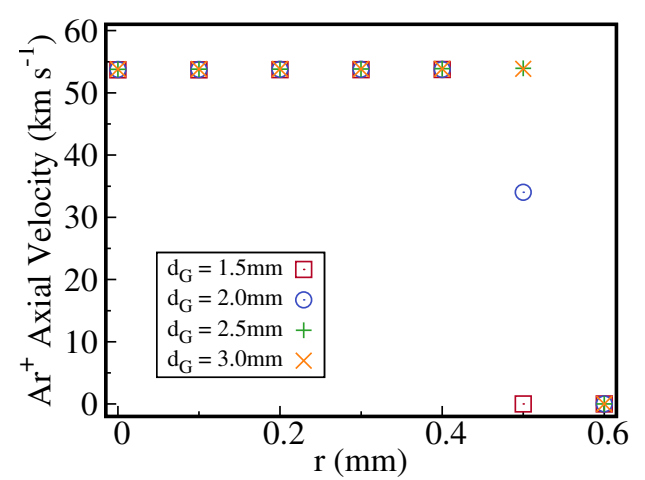

(a) Axial velocity

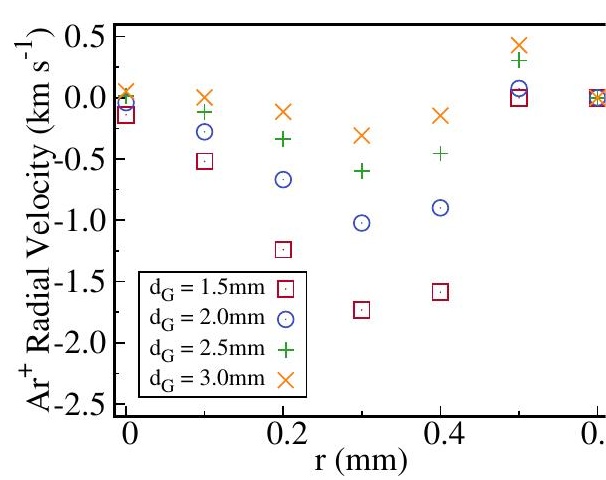

(b) Radial velocity

FIG. 7. The axial ion $v_{i, z}\left(r, z_{E}\right)$ and radial $v_{i, r}\left(r, z_{E}\right)$ velocities for different distances $d_{G}$ between the extraction and cover grids of holding constant $t_{G}=0.5 \mathrm{~mm}$. Non-zero radial velocity at the axis comes from the weighting of particles into the axis nodes.

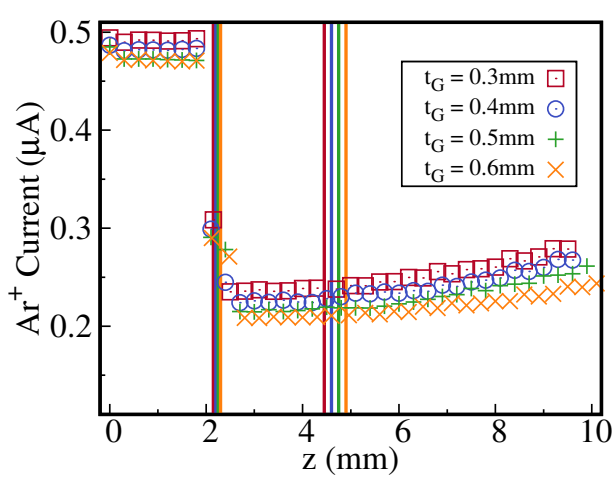

(a) Ion current $I_{i}(z)$.

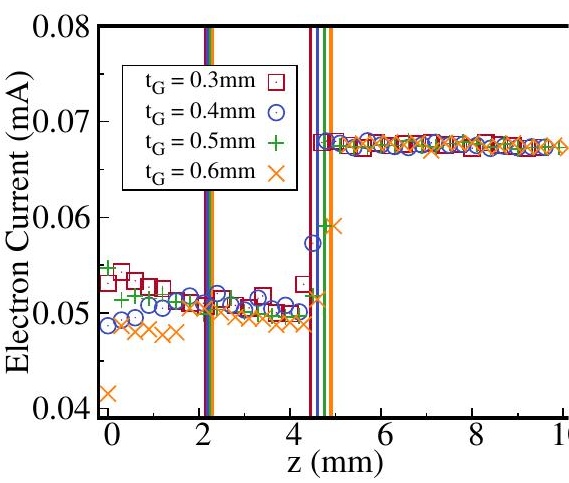

(b) Electron current $I_{e}(z)$.

FIG. 8. The currents of ions and electrons for different grid thickness $t_{G}$ of the extraction and cover grids of Fig. 2. The separation be grids remains constant to the base case of $d_{G}=2.0 \mathrm{~mm}$. Vertical lines represent the mid position of grids for each case.

by the counterflow of charges explains why reductions in the distance between the two grids have small effect in the magnitude of the ion and electron currents.

In Fig. 6 is represented the ion density radial profile $n_{i}\left(r, z_{E}\right)$ at the $z_{E}$ coordinate, corresponding to the exit plane of the cover grid for $d_{G}=1.5,2.0,2.5$ and $3.0 \mathrm{~mm}$. Its value is maximum at the axis of symmetry $r=0$ and decreases faster with the radial distance $r$ for shorter separations between the grids. Since the ion current $I_{i}(z)$ in Fig. 5a is essentially independent of the separation between grids, the exit ion beam becomes more focused as the distance $d_{G}$ reduces. Therefore, ions concentrate along the hole centerline when the distance between grids is reduced.

This ion collimation effect as $d_{G}$ decreases is also observed in Fig. 7 that shows the average axial $v_{i, z}\left(r, z_{E}\right)$ and radial $v_{i, r}\left(r, z_{E}\right)$ components of the ion velocity at the exit plane.
The axial velocity of ions in figure $7 \mathrm{a}$, within the $\mathrm{km} / \mathrm{s}$ range, are in accordance with those measured in th oratory ${ }^{7}$. They decrease faster with $r$ for smaller distan between the grids. The radial component in Fig. $7 \mathrm{~b}$ is 1 ders of magnitude lower than $v_{i, z}\left(r, z_{E}\right)$ and remains ne for most cases, except for $d_{G} \geq 2.5 \mathrm{~mm}$. The non-zero velocity at the axis comes from the weighting of particle the finite element mesh, as some may be far from the ax still deposit some information into the axis nodes. Thi in radial velocity indicates that the ion beam becomes skew.

Additionally, small changes in the thickness $t_{g}$ of gric have a low impact in the electric charge transport. Fis shows the ion $I_{i}(z)$ and electron $I_{e}(z)$ currents flowing $\mathrm{r}$ tively downstream and upstream of the simulation doma $t_{G}=0.3,0.4,0.5$ and $0.6 \mathrm{~mm}$ holding constant the sepa 


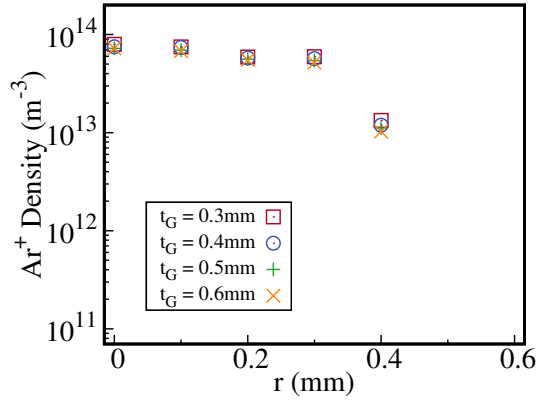

FIG. 9. The axial number density $n_{i}\left(r, z_{E}\right)$ of ions along the radial direction of Fig. 2 for different grid thickness $t_{G}$ of the extraction and cover grids, holding constant $d_{G}=2.0 \mathrm{~mm}$.

$d_{G}=2.0 \mathrm{~mm}$ between both meshes.

Increments in $I_{i}(z)$ as $t_{G}$ is reduced are small and the electron current $I_{e}(z)$ remains unaltered. Both have similar values to those of Fig. 5a and variations in the plasma density in the ionization chamber are essentially responsible for the increments in the ion current downstream the cover grid. The larger reduction observed for $t_{G}=0.6 \mathrm{~mm}$ seems to be related with the increased particle losses for thick grids.

The radial profile of ion density $n_{i}\left(r, z_{E}\right)$ at the exit plane of the cover grid in Fig. 9 is insensitive to changes in the thickness of both grids. This is also the case for the axial and radial components of the average ion velocity in Fig. 10. Thus, the ons and electron dynamics seem unaltered by grid thickness.

\section{CONCLUSIONS}

The characterization of the alphie two-grids system requires a different numerical approach than those used for ion optics of conventional GIEs. In this last case, only ions travel through the open spaces of the grids, whereas a counterflow of ions and electrons is transported through an alphie thruster. The ion current is not space-charge limited since the local electric field is self-consistently determined by the electric potential applied to the grids and the ion and electron flows.

This electric charge transport has been simulated using the fpakc particle-in-cell code where different time scales can be used for the ion and electron time evolution. This feature reduces about $40 \%$ of the the computational time and permits exploration of changes in the basic geometry of the alphie two-grids system.

Both ions and electrons are simulated from a kinetic point of view, necessary to describe the influx of high energy electrons, that is a unique characteristic of alphie thruster operation. Moreover, the code calculates the velocities and positions of both species of particles allowing a detailed analysis of the performance impact of geometrical parameters

The counterflow of positive and negative charges produces a focused ion flow along the axis of symmetry as shown by the large difference between the axial and radial compc of the ion velocity in Figs. 7 and 10. The ions are cc trated along the centerline of the simulation domain al collimation of the beam basically depends on the distan between the extraction and cover grids as shown in Figs. 9.

However, the calculations in Figs. 5 and 8 indicat small changes in the distance $d_{G}$ between the grid their thickness $t_{G}$ have minimal impact in the ion $\mathrm{c}$ $I_{i}=I_{i, z}\left(z_{E}\right)$ at the exit plane of the cover grid of Fig. 2 suming single-charged ions for simplicity, the thrust del $F=\dot{m}_{i} v_{i, z}$ where $\dot{m}_{i}=m_{i} \times I_{i} / e$ also will be essentially stant for small changes in $d_{G}$ and $t_{G}$.

We can conclude that the alphie two-grids thruster cc is robust. Small variations in the distance between the and their thickness have little impact in both, the exhau beam and the inflow of ionizing electrons from the ca Therefore, it is potentially insensitive to moderate mo tions of its basic geometry originated by the unavoidable during long term thruster operation.

The ion current and beam collimation are not signifi affected by changes in the grid thickness. Therefore parameter can be selected according to other consider such as their mechanical characteristics or thruster facture. In addition, the spacing between the alphie is longer than those of conventional GIEs (usually a $1 \mathrm{~mm}$ ), which reduces the chances of sparks and ele breakdowns between them.

The results of Figs. 7 and 10 show that the axial ve of ions, directly related with the specific impulse and is essentially unaltered by small geometrical variations alphie two-grids system. This hints that small deform or erosion of the grids by sputtering will have a lesser $i$ in the thruster performance, unlike classical GIEs. Ho this issue requires of further numerical analysis and end laboratory tests.

\section{ACKNOWLEDGMENTS}

This work was partially funded by the Ministerio de cia, Innovación y Universidades of Spain under Grant ber RT2018-094409-B-100 and also by the Russian Sci Foundation under Grant number 21-72-20029.

\section{DATA AVAILABILITY}

The data that support the findings of this study are av: from the corresponding author upon reasonable request

${ }^{1}$ D. M. Goebel and I. Katz, Fundamentals of electric propulsion. Hall thrusters (John Wiley \& Sons, Hoboken, New Jersey, USA, 20 ${ }^{2}$ S. Mazouffre, Plasma Sour. Sci. Technol. 25, 033002 (2016).

${ }^{3}$ I. Levchenko, S. Xu, S. Mazouffre, D. Lev, D. Pedrini, D. Goebel, rigues, F. Taccogna, and K. Bazaka, Phys. Plasmas 27, 020601 (20 ${ }^{4}$ M. Sangregorio, X. K., N. Wang, N. Guo, and Z. Zhang, Chinese J naut. 31, 1635 (2018). 


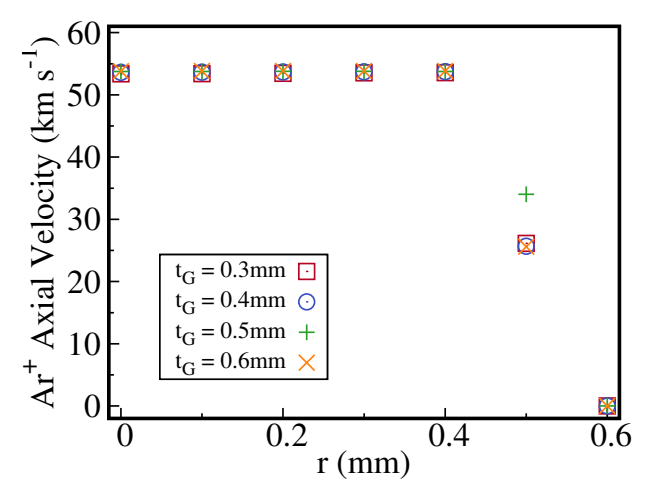

(a) Axial velocity.

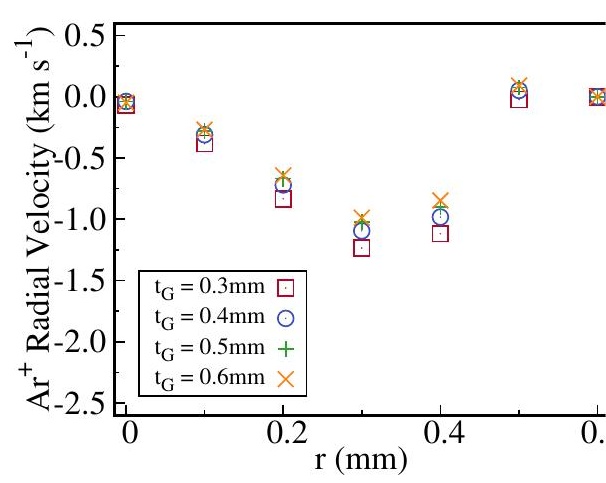

(b) Radial velocity.

FIG. 10. The axial ion $v_{i, z}\left(r, z_{E}\right)$ and radial $v_{i, r}\left(r, z_{E}\right)$ velocities for different grid thickness $t_{G}$ of the extraction and cover grids, holding ce $d_{G}=2.0 \mathrm{~mm}$.

${ }^{5}$ L. Conde, J. L. Domenech-Garret, J. M. Donoso, E. Del Río, and M. A. Castillo, "Plasma accelerator with modulated thrust," (2019), US Patent No. $10,172,227$ B2.

${ }^{6}$ L. Conde, J. L. Domenech-Garret, J. M. Donoso, E. Del Río, and M. A. Castillo, "Plasma accelerator with modulated thrust and space born vehicle with the same," (2015), EPO Patent No. EP3369294B1.

${ }^{7}$ L. Conde, J. Domenech-Garret, J. Donoso, J. Damba, S. Tierno, E. Alamillo-Gamboa, and M. Castillo, Phys. Plasmas 24, 123514 (2017).

${ }^{8}$ L. Conde, J. González, J. Donoso, J. Domenech-Garret, J. Damba, P. Maldonado, J. Grabulosa, and M. Lahoz, in Proceedings of the 36th International Electric Propulsion Conference (Vienna, Austria, 2019) pp. 1-9, IEPC paper 2019-A-643.

${ }^{9}$ J. González and L. Conde, Phys. Plasmas 26, 043505 (2019).
${ }^{10}$ D. Dyubo and O. Y. Tsybin, St. Petersburg Polytecnical State Un Journal. Physics and Mathematics 13, 78 (2020).

${ }^{11}$ S. Cho, H. Watanabe, K. Kubota, S. Iihara, K. Fuchigami, K. Uemat I. Funaki, Phys. Plasmas 22, 103523 (2015).

${ }^{12}$ I. Hutchinson, Phys. Plasmas 24, 055601 (2017).

${ }^{13}$ S. Hosseini Jenab and F. Spanier, Phys. Rev. E 95, 053201 (2017)

${ }^{14}$ H. Kim, F. Iza, S. Yang, M. Radmilović-Radjenović, and J. Lee, D: Appl. Phys. 28, R283 (2005).

${ }^{15}$ V. Kobolob and R. Arslanbekov, J. Phys. Conf. Ser. 719, 012020 ${ }^{16}$ D. Tskhakaya, K. Matyash, R. Schneider, and F. Taccogna, Contrib. Phys. 47, 563 (2007)

${ }^{17}$ L. Liu, W. Zou, H. Wang, F. Guo, and D. Liu, Phys. Plasmas 25, (2018). 


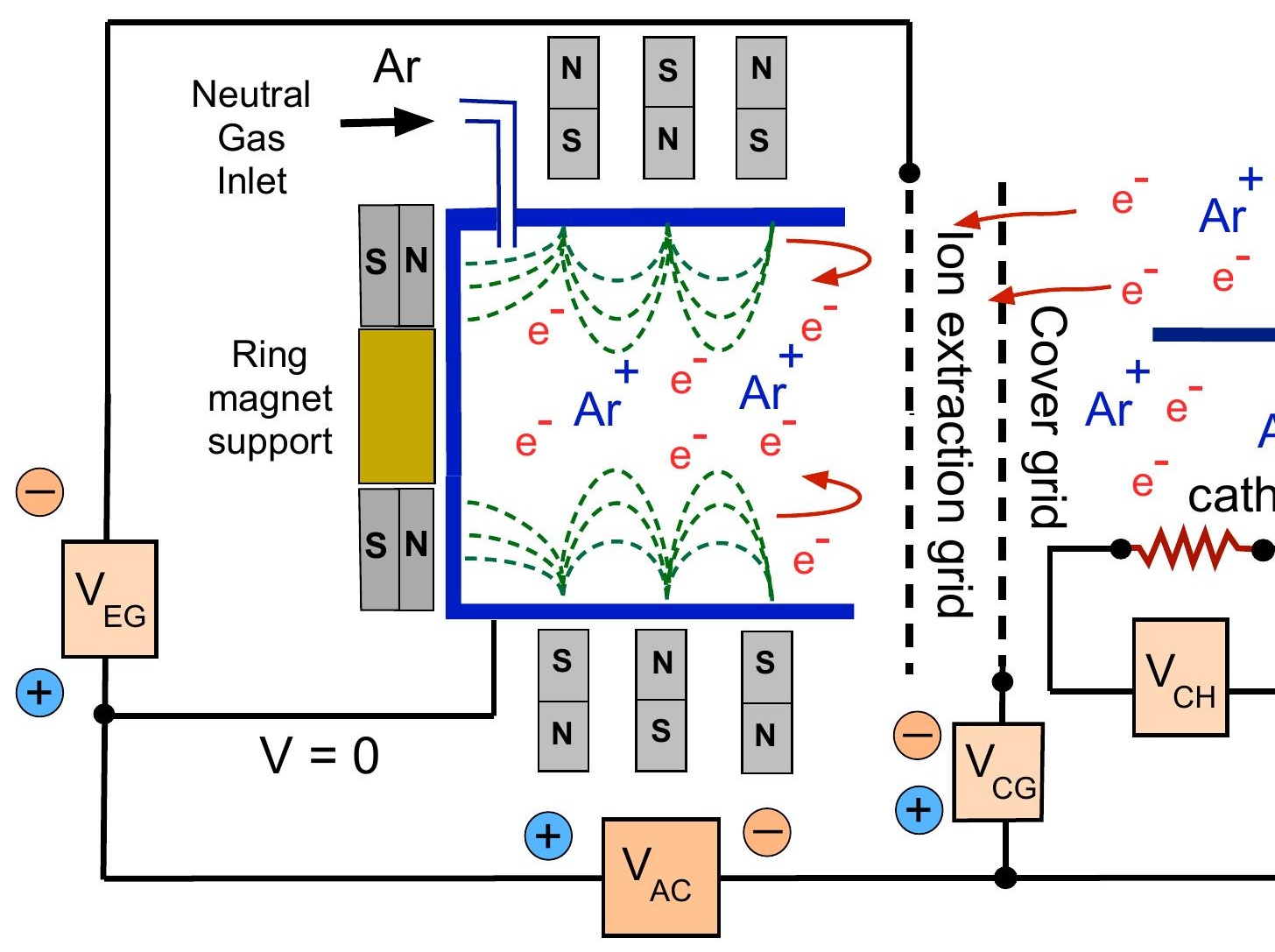




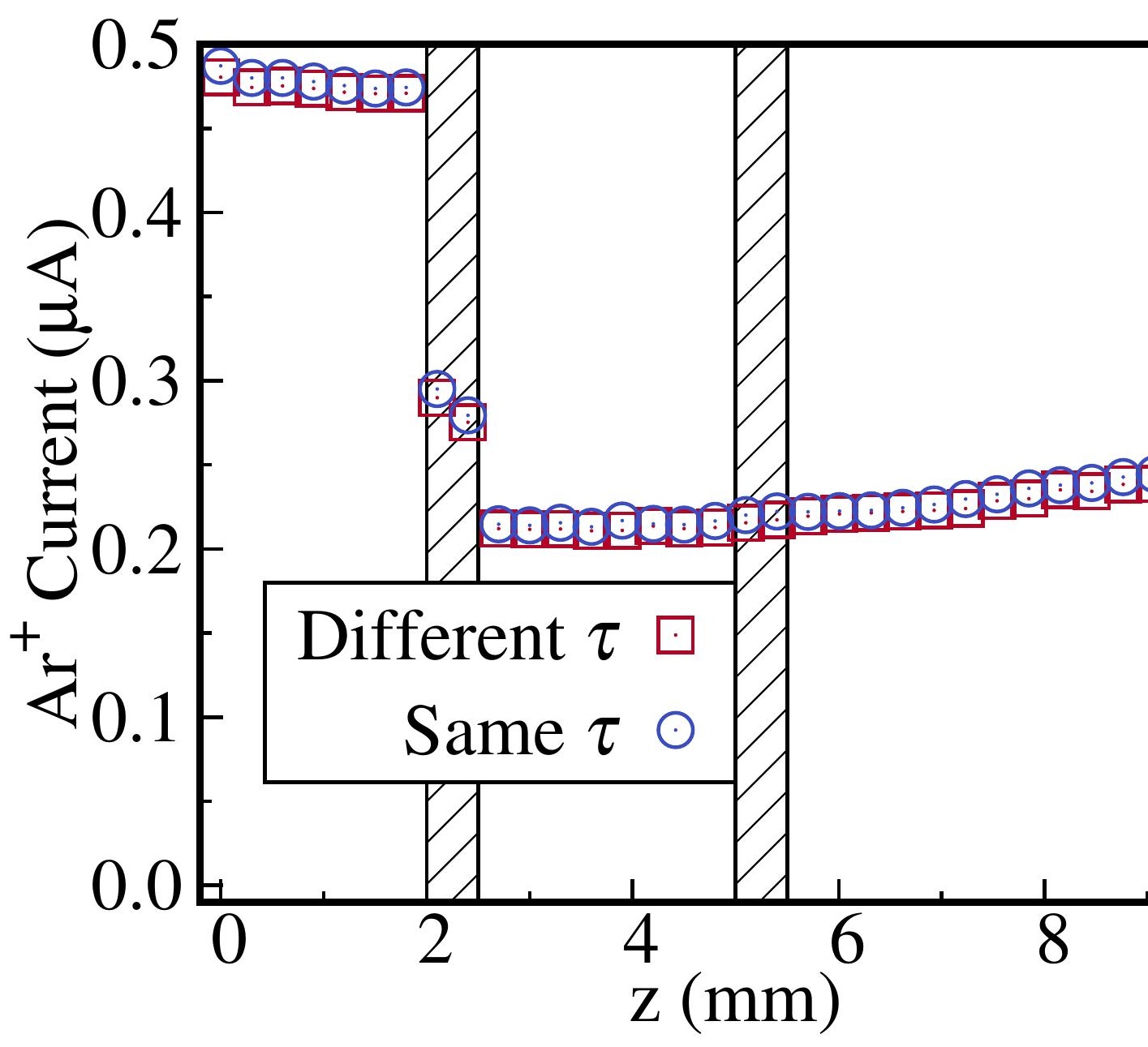




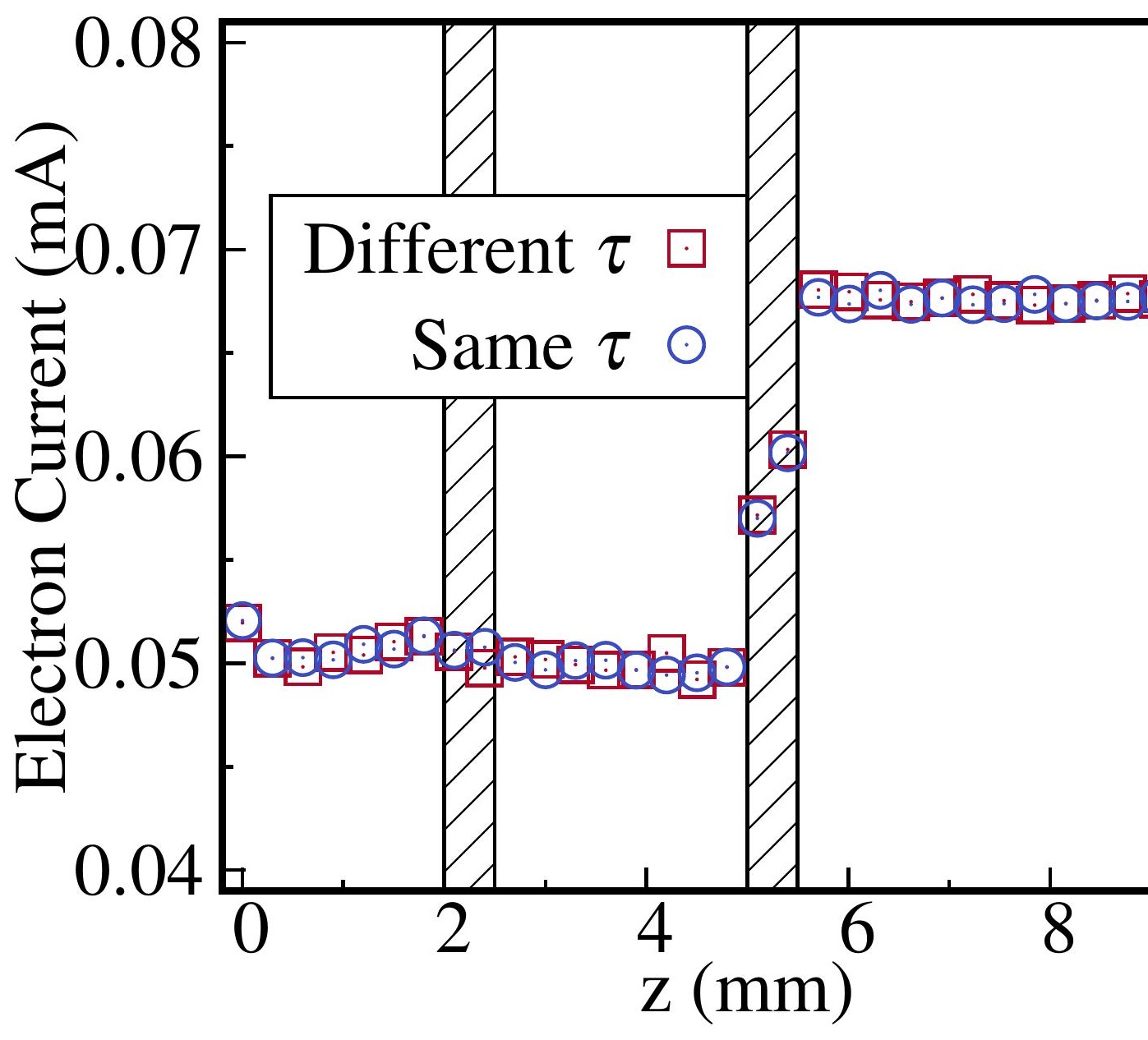




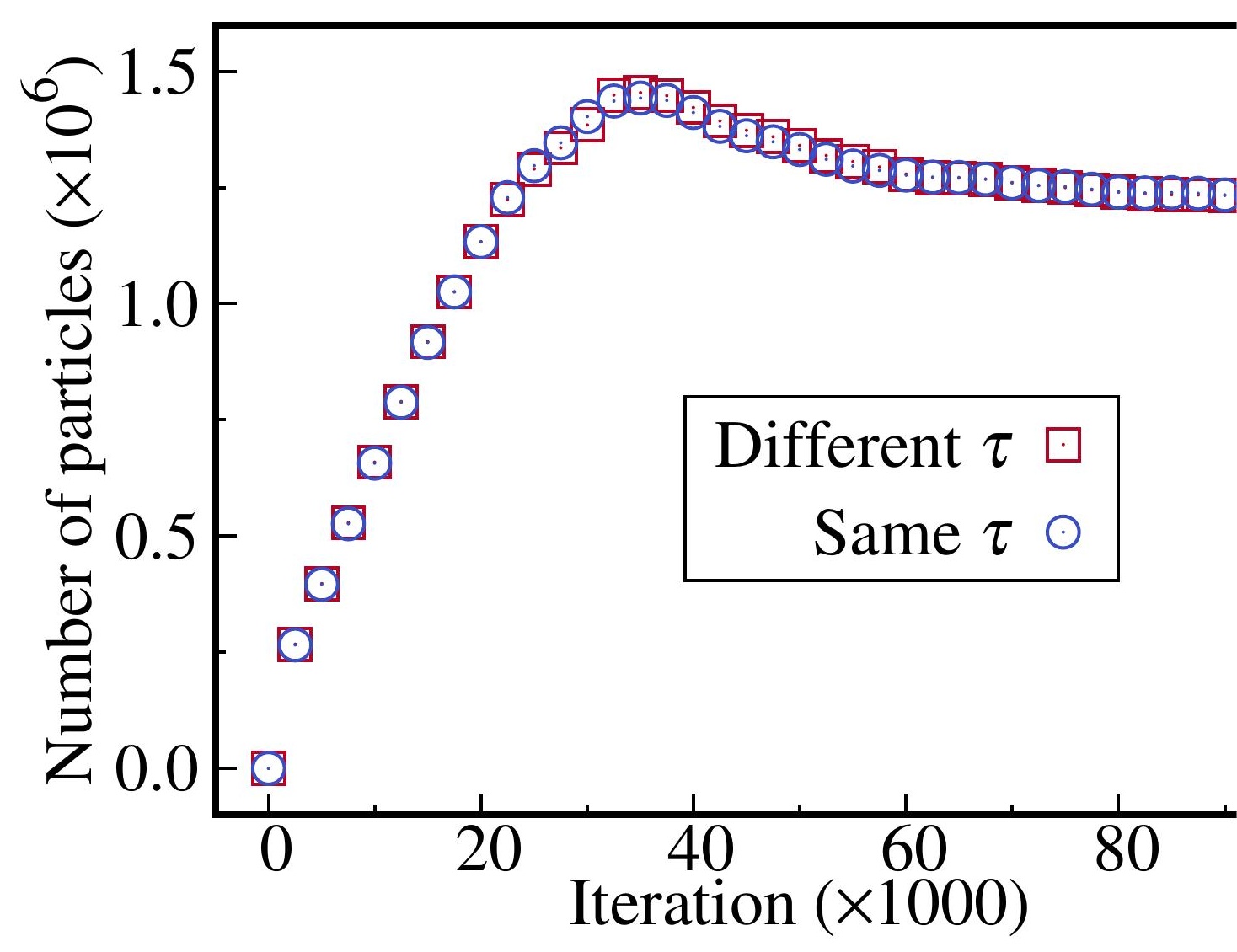




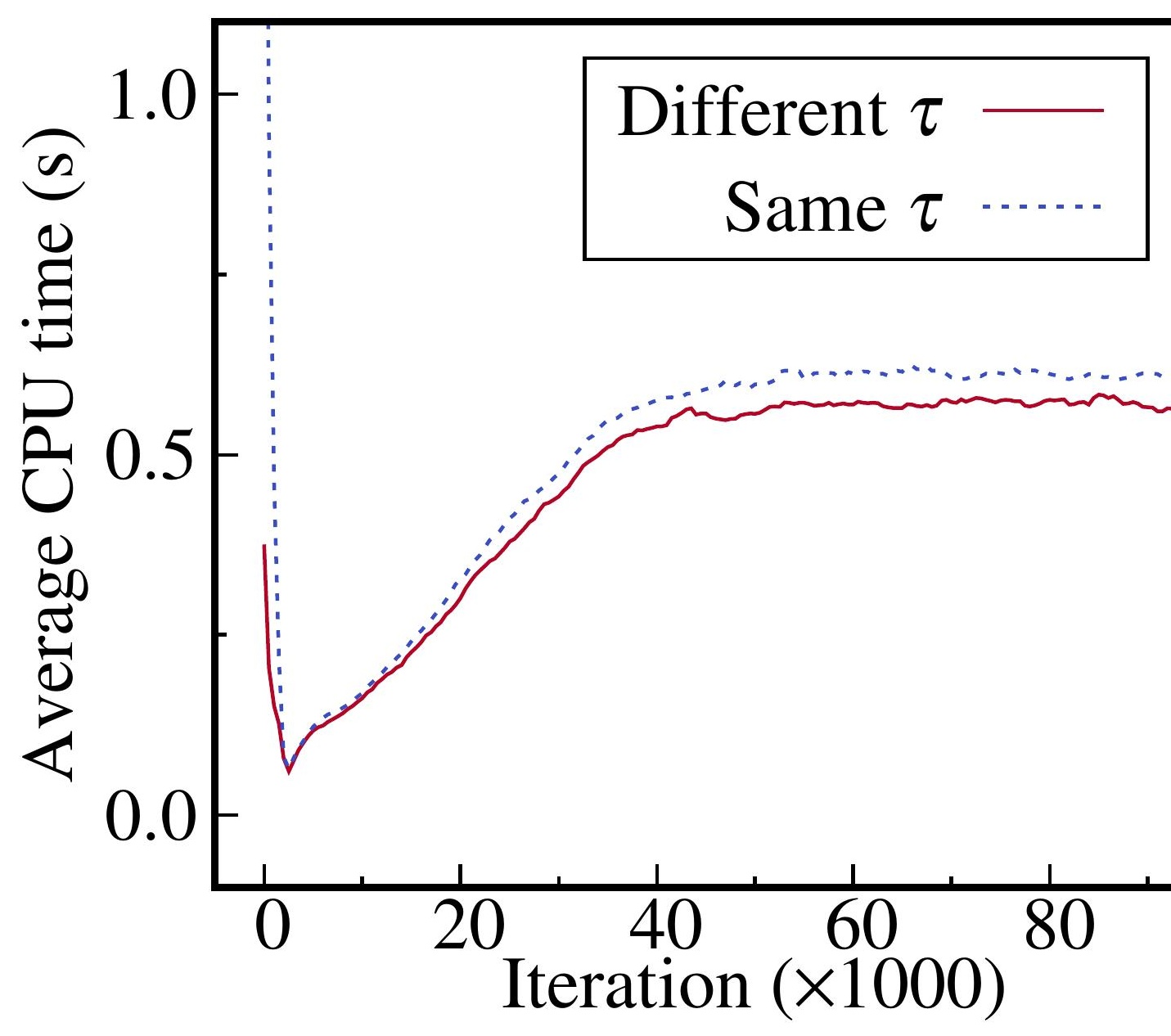




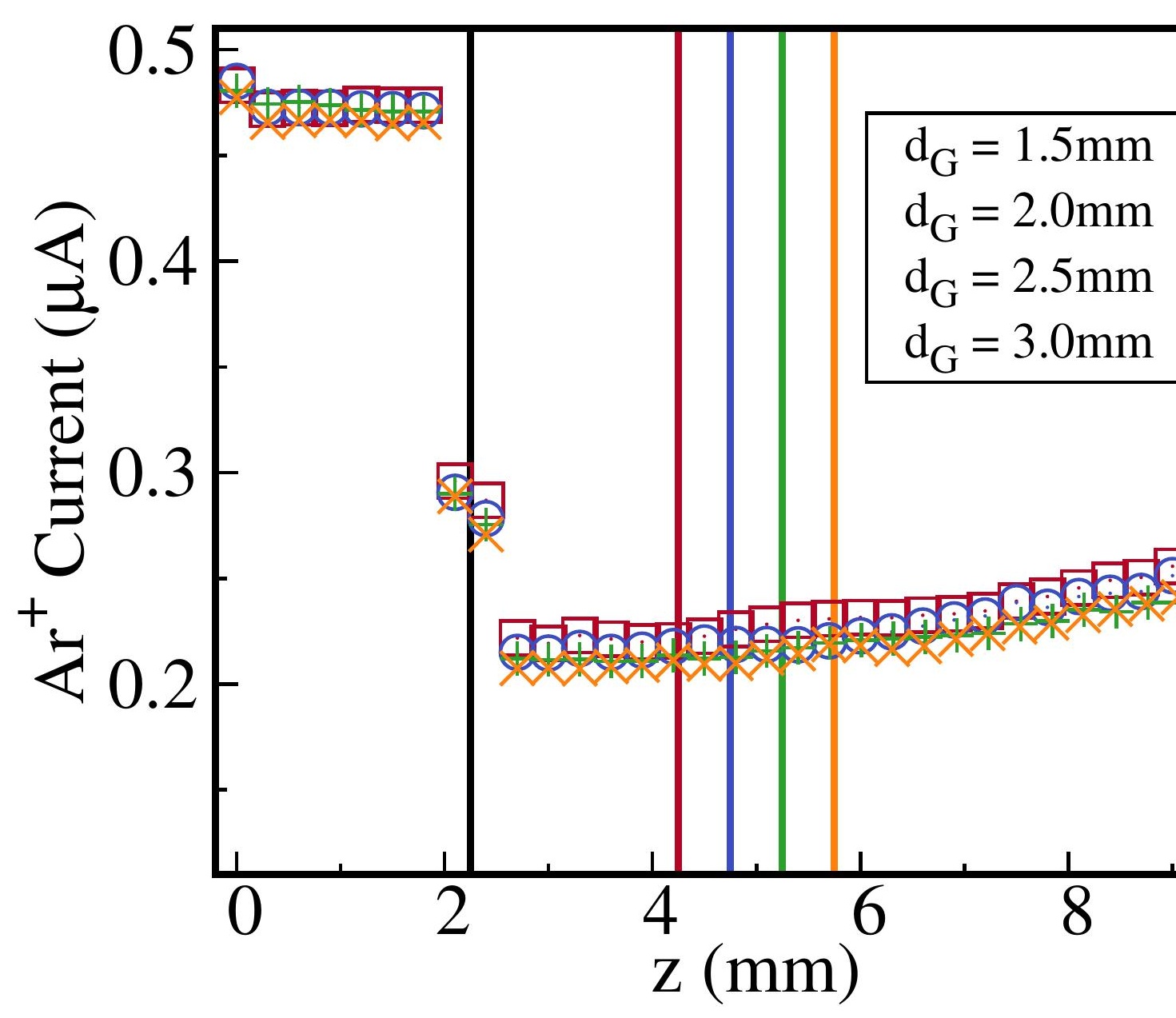




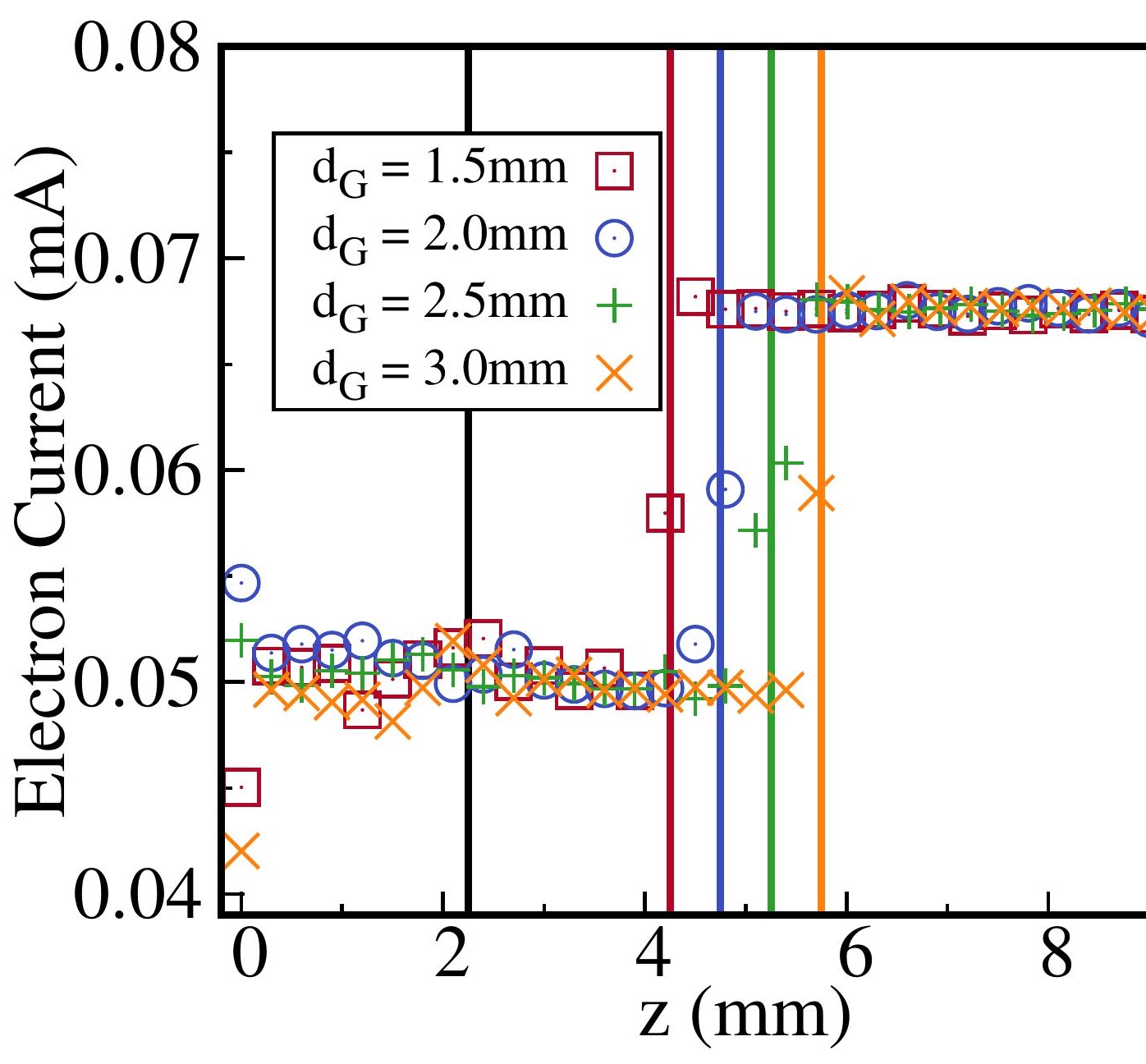




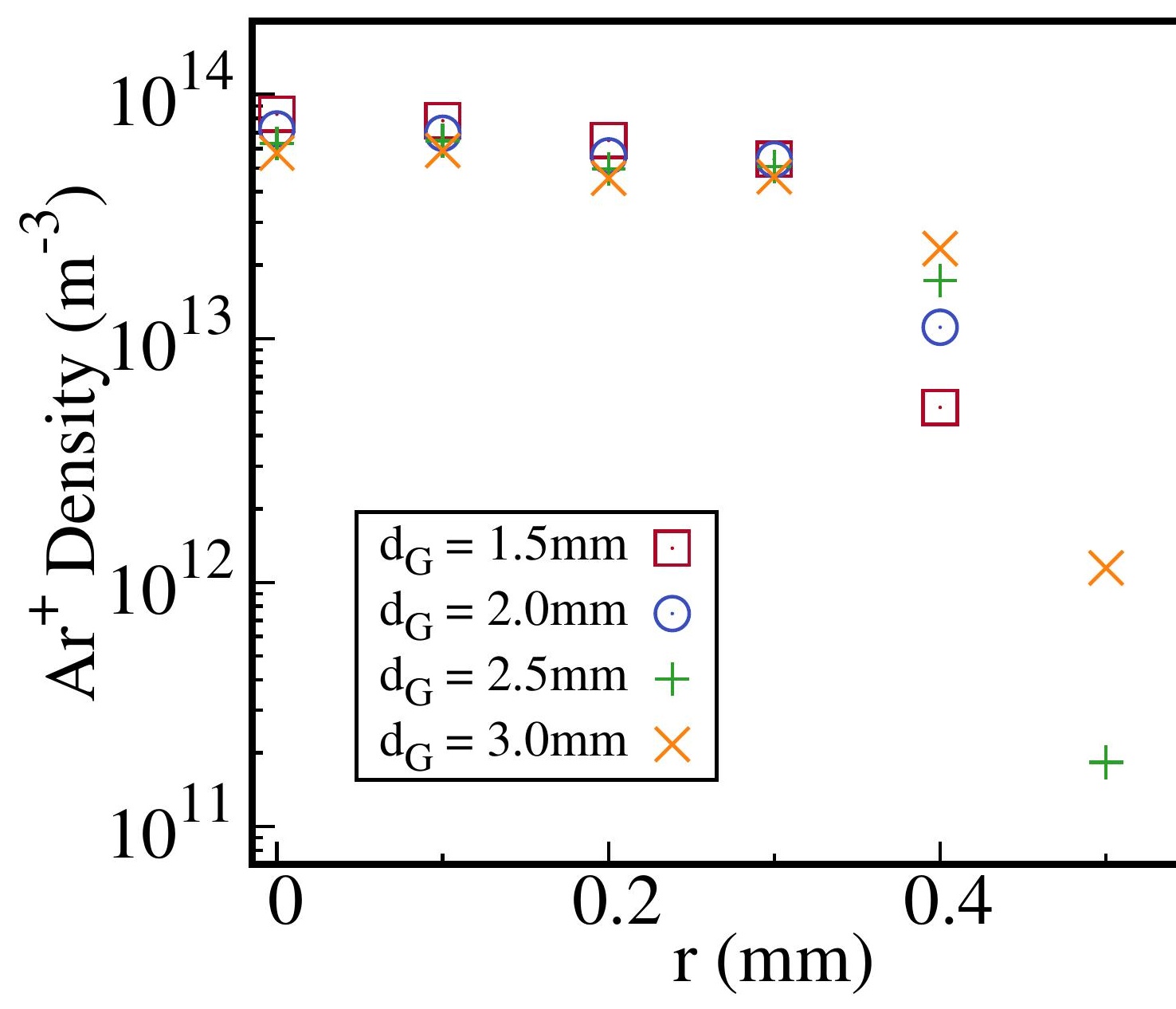




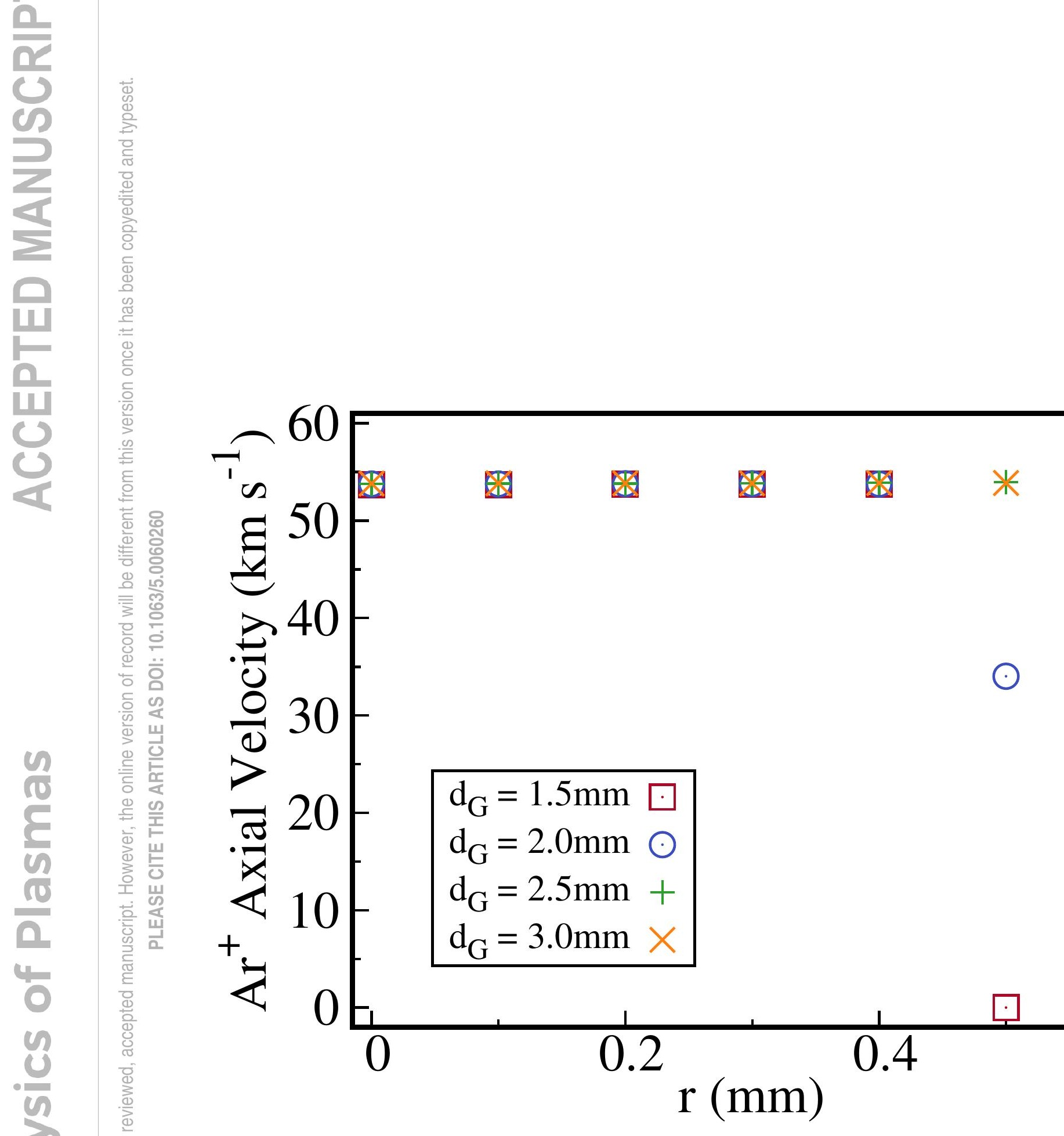




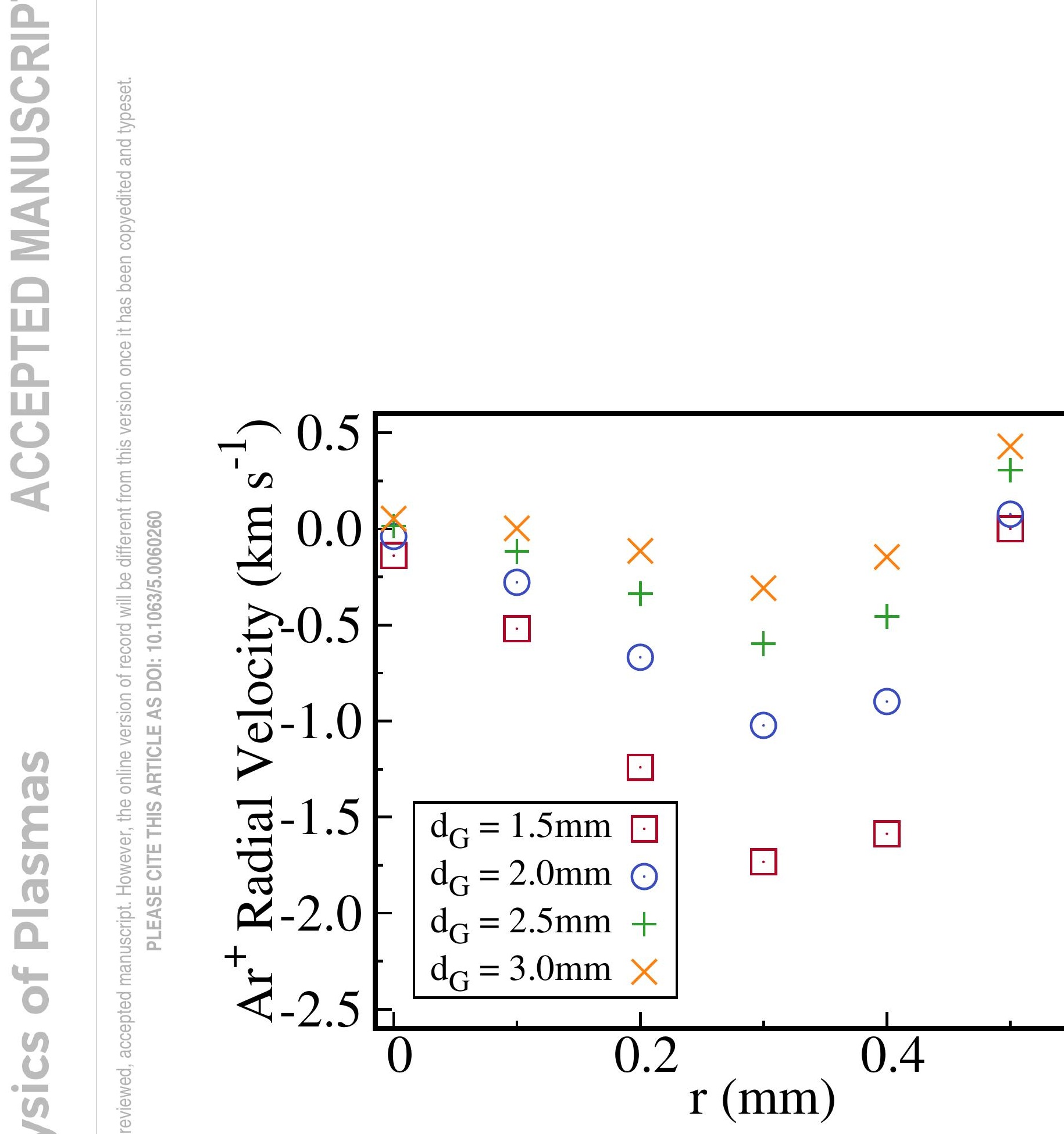




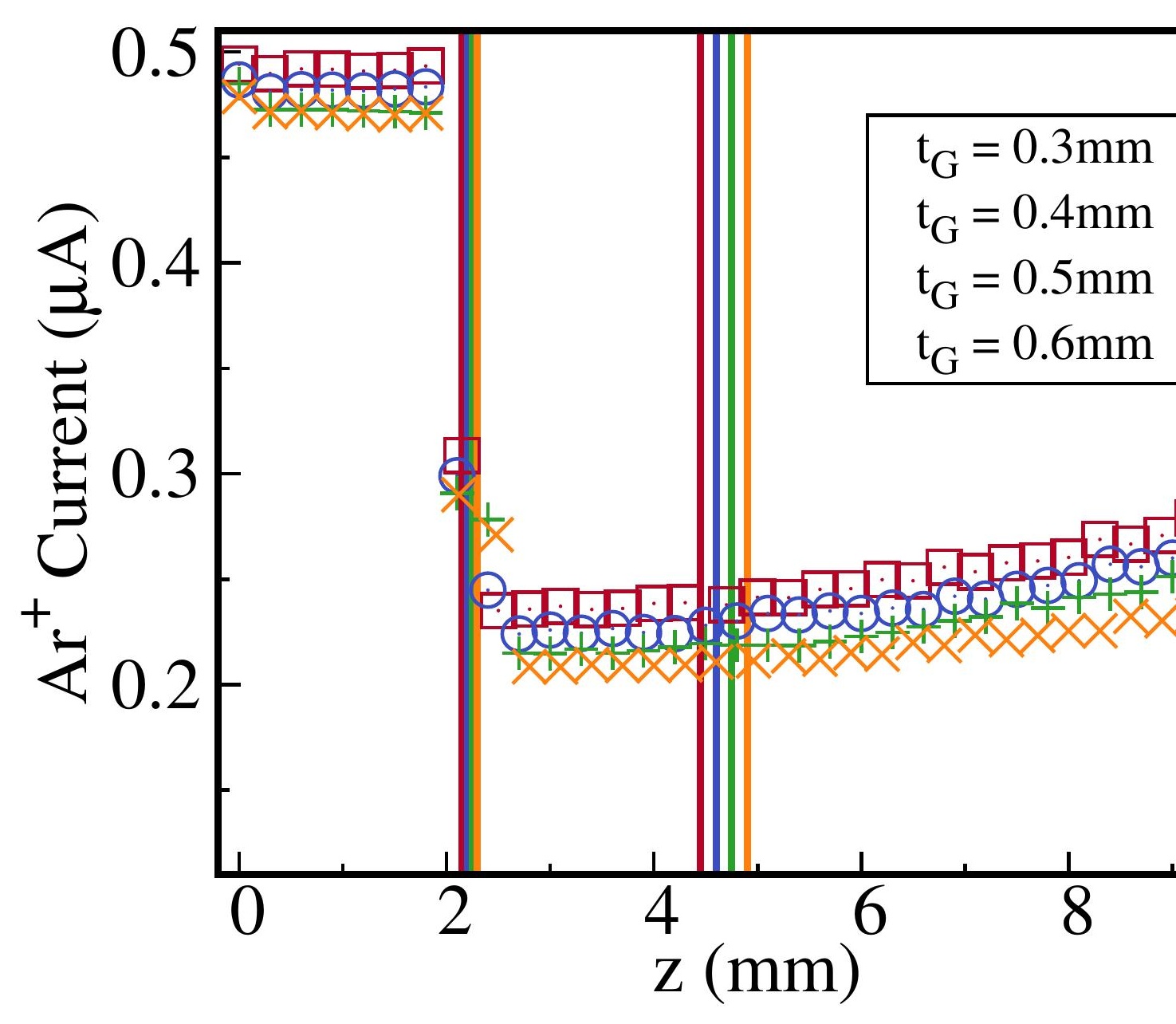




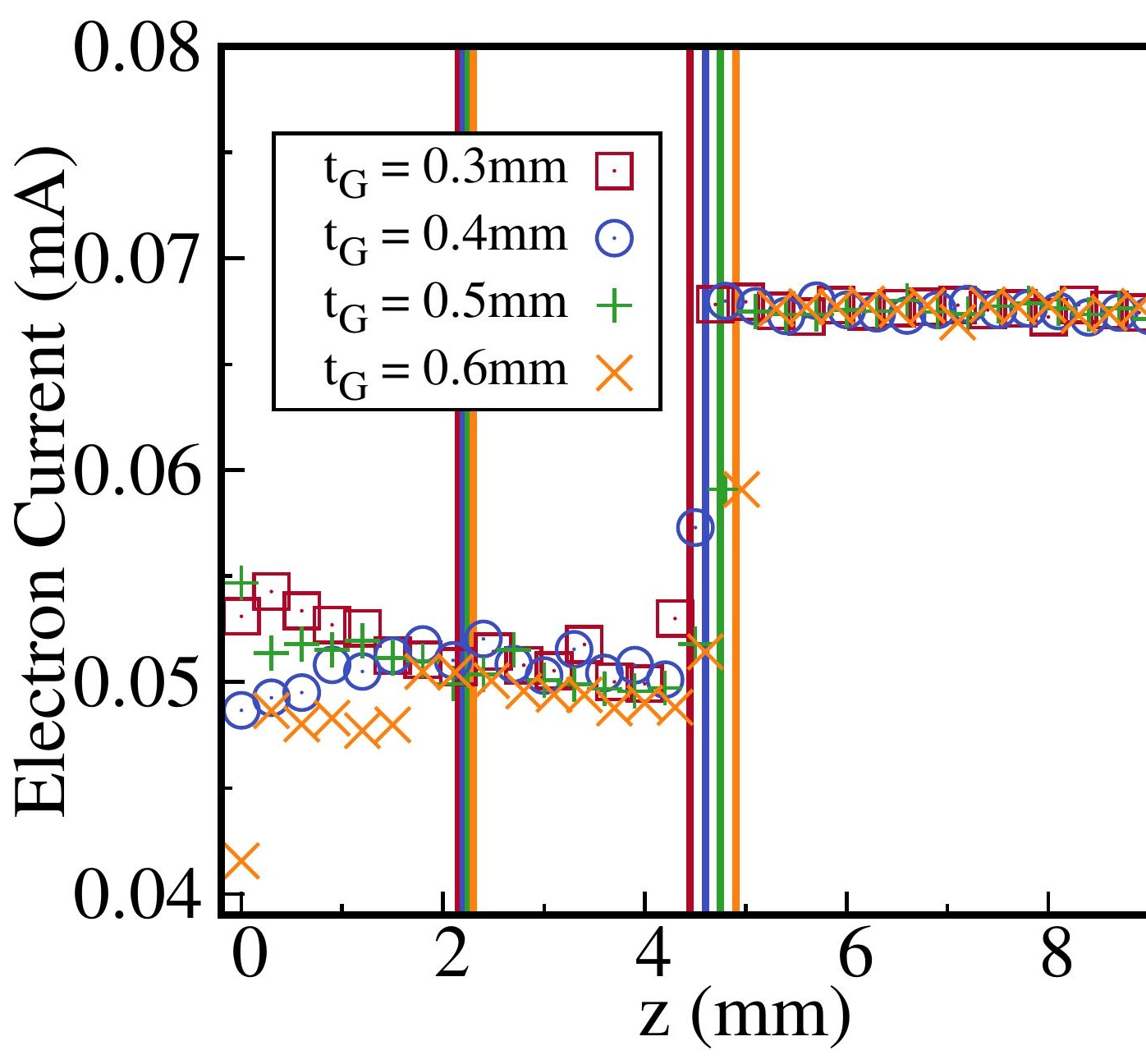




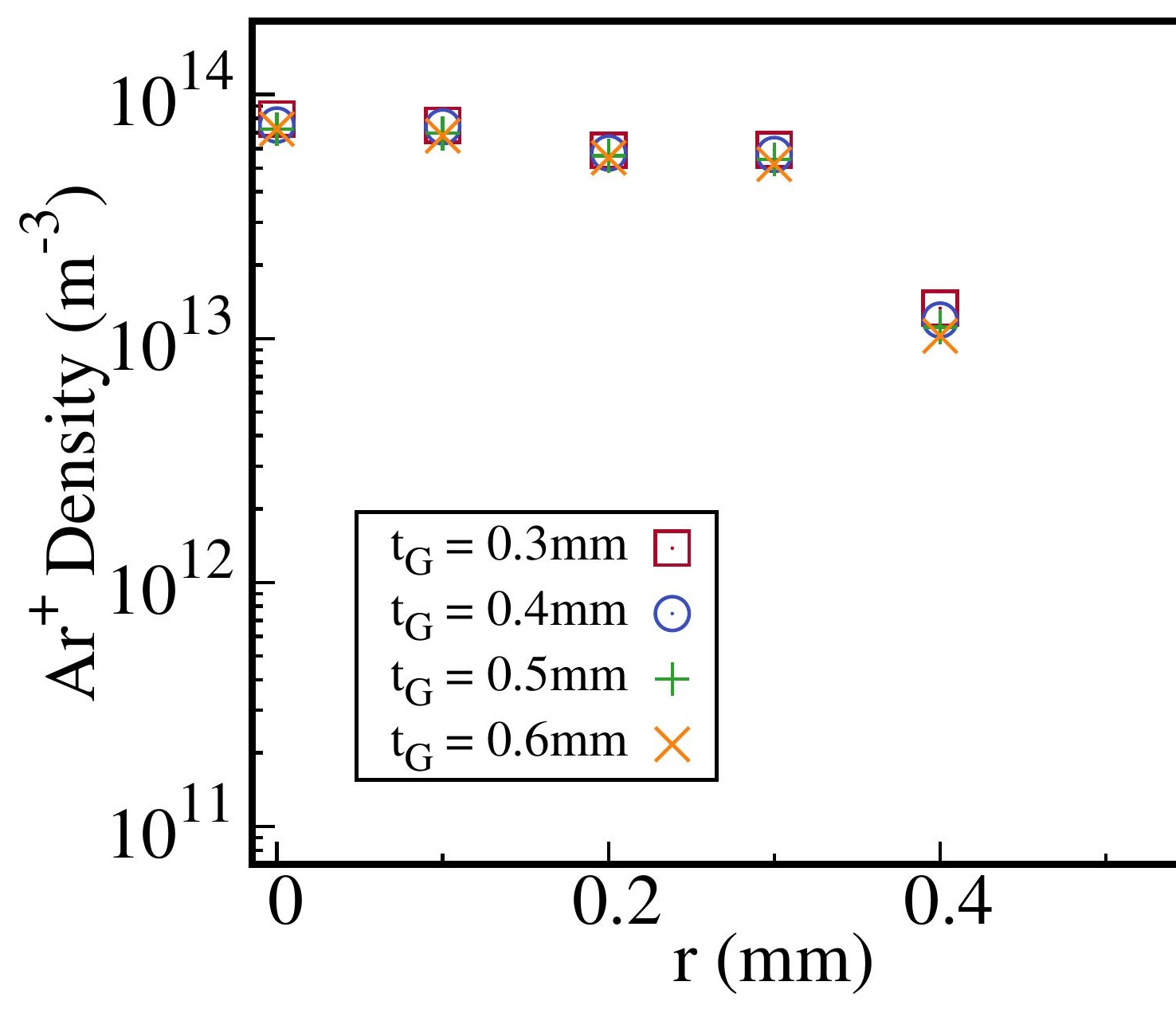




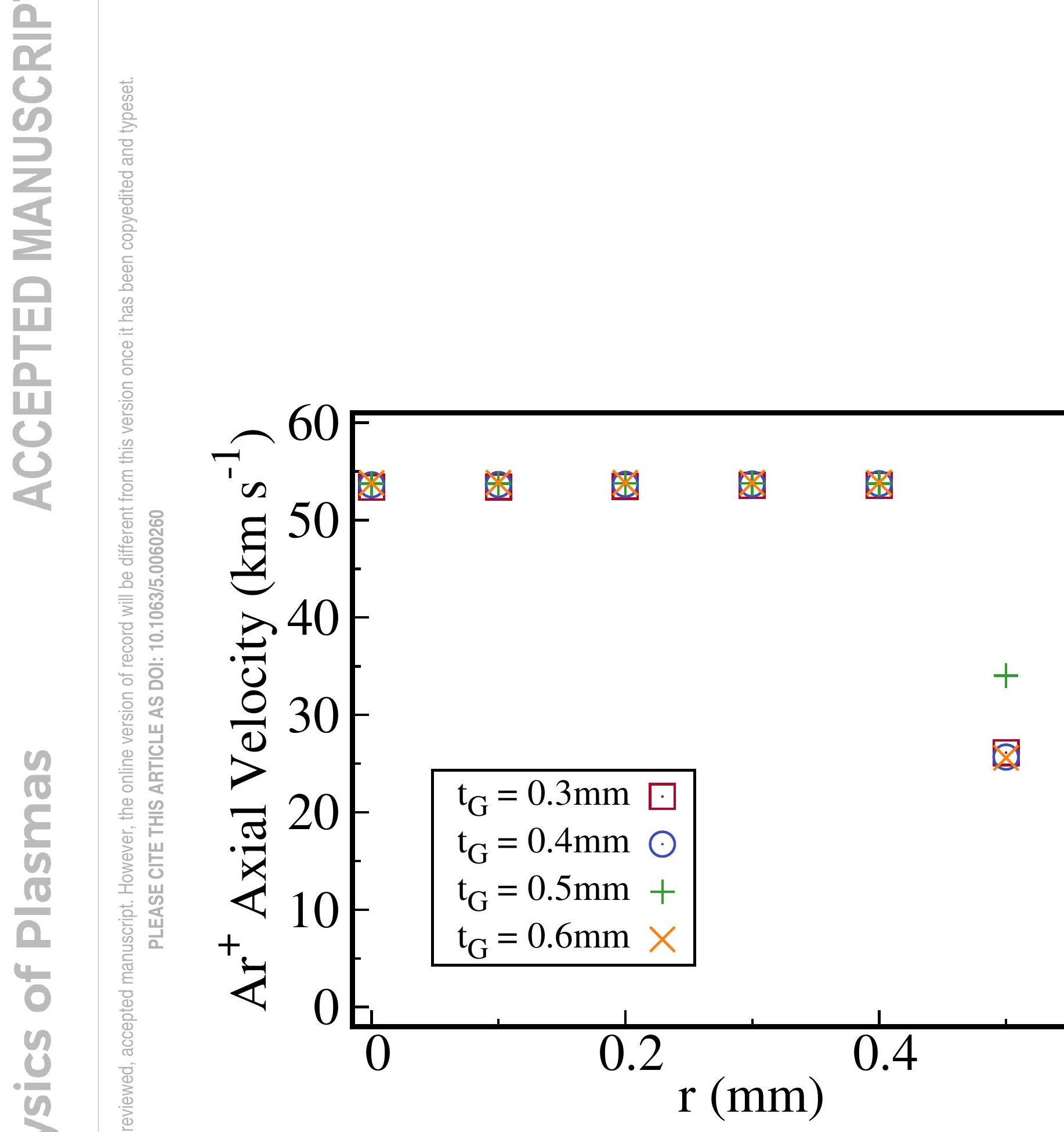




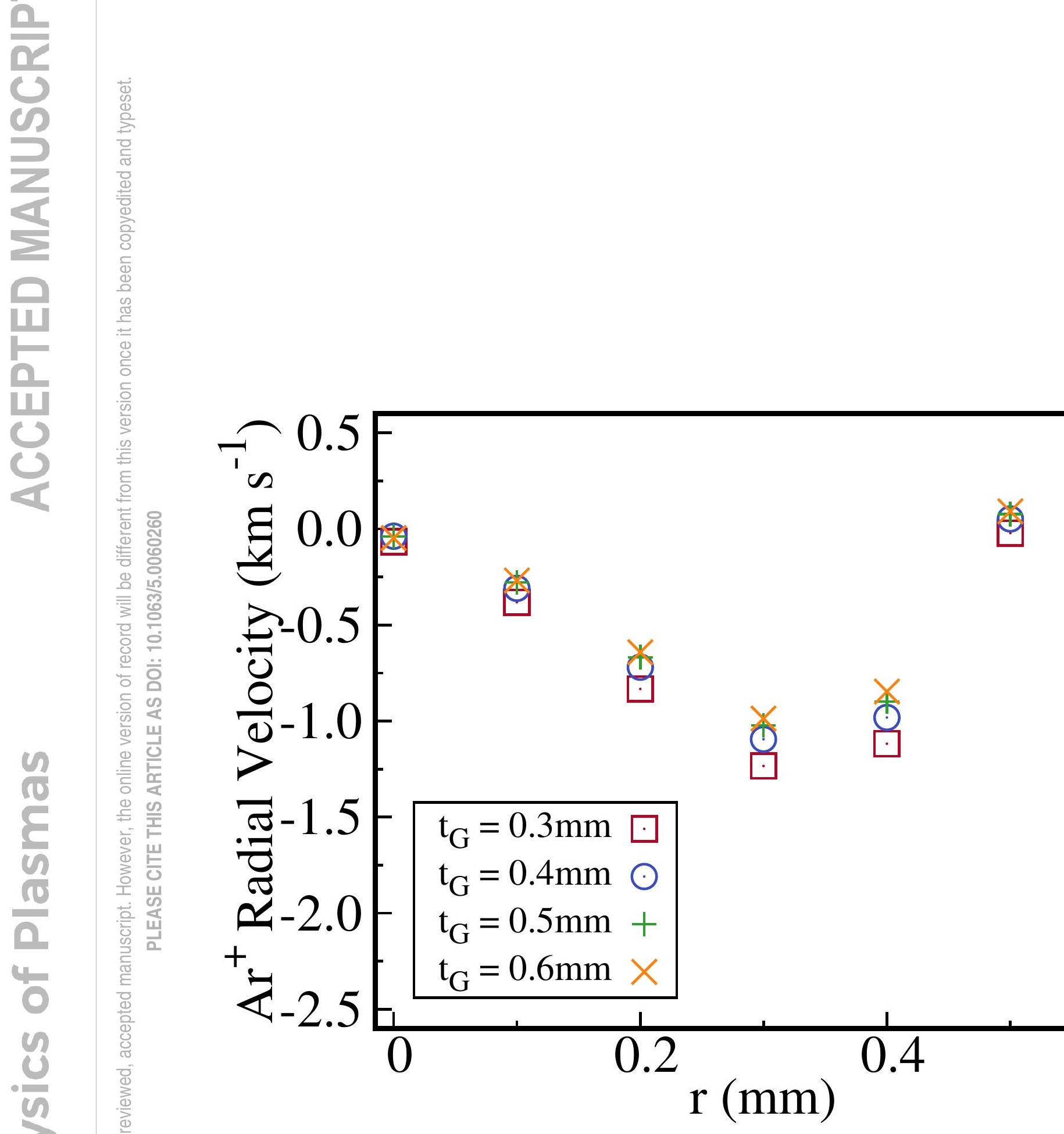

